\title{
Design opportunities for supporting informal caregivers
}

\author{
Author(s) \\ Bosch, Lilian; Kanis, Marije \\ DOI \\ 10.1145/2851581.2892354
}

Publication date

2016

Document Version

Final published version

Published in

Proceedings of the $2016 \mathrm{CHI}$ Conference Extended Abstracts on Human Factors in Computing Systems

Link to publication

Citation for published version (APA):

Bosch, L., \& Kanis, M. (2016). Design opportunities for supporting informal caregivers. In J. Kaye, \& A. Druin (Eds.), Proceedings of the 2016 CHI Conference Extended Abstracts on Human Factors in Computing Systems (pp. 2790-2797). Association for Computing Machinery. https://doi.org/10.1145/2851581.2892354

It is not permitted to download or to forward/distribute the text or part of it without the consent of the author(s) and/or copyright holder(s), other than for strictly personal, individual use, unless the work is under an open content license (like Creative Commons).

If you believe that digital publication of certain material infringes any of your rights or (privacy) interests,

please let the Library know, stating your reasons. In case of a legitimate complaint, the Library will make the material inaccessible and/or remove it from the website. Please contact the library:

https://www.amsterdamuas.com/library/contact/questions, or send a letter to: University Library (Library of the University of Amsterdam and Amsterdam University of Applied Sciences), Secretariat, Singel 425, 1012 WP Amsterdam, The Netherlands. You will be contacted as soon as possible. 


\section{Design Opportunities for Supporting Informal Caregivers}

\section{Lilian Bosch}

Amsterdam University of Applied Sciences

Wibautstraat 2-4

1091 GM, Amsterdam

l.b.j.bosch@hva.nl

\section{Marije Kanis}

Amsterdam University of Applied Sciences

Wibautstraat 2-4

1091 GM, Amsterdam

m.kanis@hva.nl
Permission to make digital or hard copies of part or all of this work for personal or classroom use is granted without fee provided that copies are not made or distributed for profit or commercial advantage and that copies bear this notice and the full citation on the first page. Copyrights for third-party components of this work must be honored. For all other uses, contact the Owner/Author.

Copyright is held by the owner/author(s).

CHI'16 Extended Abstracts, May 07-12, 2016, San Jose, CA, USA ACM 978-1-4503-4082-3/16/05

http://dx.doi.org/10.1145/2851581.2892354

\begin{abstract}
This paper highlights key opportunities for technology design for informal caregivers who provide long-term in-home care. For this purpose, a study with informal caregivers was conducted, including interviews $(N=4)$ and online questionnaires $(N=34)$ based on holistic analysis of supportive technologies. These

investigations provide a deeper understanding of the key opportunities in the design of technologies to support the caregiver, namely (1) making caregivers better informed and more aware of existing solutions

(2) increasing awareness of the caregivers' own wellness; (3) cherishing the valuable, positive moments of caregiving (e.g. by capturing precious moments) and (4) encouraging meaningful social interactions among caregivers for strengthening social ties.
\end{abstract}

\section{Author Keywords}

Human-centered design; informal caregivers; home care; assistive technologies; health and wellness.

\section{ACM Classification Keywords}

H.5.m. Information interfaces and presentation. HCI Miscellaneous; J.3 Life and Medical Sciences, Health; K.4.2 Social Issues 


\section{Introduction}

Long-term care for older adults and people with (chronic) illness or disabilities is increasingly being provided in-home, instead of in institutions $[10,12]$. As a result, there is a large and growing number of informal caregivers [12]; people (e.g. family members, friends or neighbors, and often women $[12,31]$ ) providing the needed long-term (in-home) care as nonprofessionals for their loved one. The extent of required assistance from an informal caregiver depends on the care recipients' needs and the amount of available informal- and professional help (e.g. nurses). Their workload can vary from help with some household chores, to daily total and complex care (such as bathing, administering medications or dressing wounds), when a care recipient is fully dependent on his or her caregivers. As informal caregivers have become imperative to (home) care provision, the impact of caregiving on informal caregivers' health and caregiver burden have come to be well-studied topics. Over time, caregiving can take a toll on informal caregivers emotionally $[1,17,25]$, physically $[1,25]$ and socially $[1,3]$.

\section{Solutions for caregiving}

We have identified various non-digital support efforts to help and unburden informal caregivers, such as informal care organizations (that provide information and training), respite care (temporary relieve of care responsibilities by transferring it to others), social support (e.g. support group meetings) and services to help decrease the task load (e.g. household help or meal delivery services). In technology-related fields such as Human Computer Interaction (HCI) and information and communication technologies (ICT) there has also been a growing interest in solutions for the context of in-home care (e.g. (sensor) monitoring solutions $[15,18,28-30]$, self-management of health for patients $[2,19,21]$, assistive technologies for the home $[8,9,13]$ and coordination of care amongst caregivers $[4,5,22])$. However, in general, the vast majority of such research still largely focuses on technological solutions that are patient-centric, while aiming to help provide more efficient or effective care, and do not particularly prioritize the caregivers' needs and their wellbeing. Some researchers [6] argue that systems and tools for in-home care and caregiving -particularly the ones focusing on providing efficient patient carecan even add to the burden that a caregiver experiences. Also, such research (and its solutions) is often aimed towards caregivers of a specific patient group (e.g. ageing [6,22], Alzheimer's disease $[20,24,27]$ or depression [32]) and not so much on caregivers as a group in general. Moreover, most studies have focused on the negative aspects of caregiving, such as less life satisfaction and depression [1]. Nonetheless, there are also positive (emotional) sides to providing informal care such as companionship, fulfilment, love and personal development $[7,25,26]$ that deserve more highlighting. Therefore, although ICT are increasingly being explored as a possible solution for health care related issues such as in-home care, these predominantly focus on illness rather than wellness [14]. The challenge in the context of HCI and informal care is to design technologies that are truly desired and helpful for its users [22]. Consequently, this work particularly aims to increase understanding of the needs of informal caregivers in general and assesses their attitudes towards current and potential technological solutions. The contribution of this work is the identification of opportunities for the design of 


\section{Scenarios}

An overview of the scenario solutions that were proposed to caregivers $(N=38)$, ranked by mean score of

appreciation on a five-point scale ( $1=$ poor, $5=$ excellent).

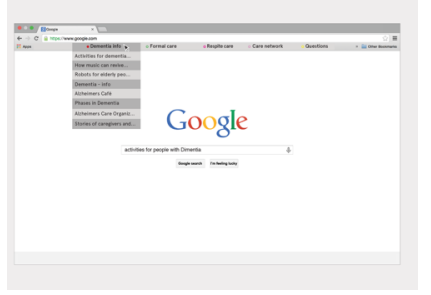

1. Information tool

(Mean=3.9 / SD=1.3 / used by $61 \%$ ). An online tool to search and collect quality information about the health concerns of the care recipient.

\section{Caregiver relaxation}

(Mean=3.7 / SD=1.4 / used by $42 \%$ ). An application to encourage caregivers to have an occasional break during care provision for relaxation and amusement.

\section{Online support group} (Mean $=3.6 / \mathrm{SD}=1.4 /$ used by $63 \%)$. An online support group to share stories,

knowledge and advice among caregivers. technologies that particularly support the wellness of informal caregivers from a holistic perspective.

\section{Study approach}

Several activities were carried out to gain a deeper understanding of caregivers and to identify opportunities for technological solutions to help support their needs, namely:

1) First, an analysis of current (technological) solutions for in-home care was conducted, by searching for papers on home care technologies in the ACM database (using keywords such as home care, (informal) caregiver, family carer and assistive technologies) and investigating commercially available systems and solutions (e.g. by visiting industrial fairs). Affinity diagramming [16] was used to cluster these different types of technologies. Based on this analysis, a set of 17 scenarios for technological solutions to support caregivers was drafted, as shown in the sidebar on page 3-6. These scenarios represent different perspectives and themes of caregiver supporting technologies and were presented in questionnaire form to all of the participants ( $N=38: 4$ during interviews and 34 through an online questionnaire). For each solution scenario that was shown, every participant valued the solution on an incremental 5-point appreciation scale ( $1=$ poor, $5=$ excellent) and indicated whether or not they currently used a similar solution. Obviously, the proposed solution and how it is valued depends on how it is exactly designed and interacted with. However, this method was chosen to first carefully establish users' general attitudes towards a large group of existing and potential technologies at early stage.
2) Semi-structured interviews were conducted with informal caregivers $(N=4)$. Each session took about 1.5 hours and was audio-recorded and transcribed for analysis afterwards. During the interviews, the researchers focused on identifying problems that the informal caregivers faced in the context of their care situation, on positive coping strategies that they applied, and on discussing their current technology usage and desires. To measure the informal caregivers' perceived burden, the 13-item Caregiver Strain Index (CSI) [23] was used during each interview. The care situations and health concerns of the care recipients of the study population were diverse, yet all caregivers provided care to someone in-home in or close to Amsterdam.

3) To compliment the interview study and increase understanding of caregiver needs from a broader group of participants, an online questionnaire was distributed through Dutch and English Facebook support groups to informal caregivers of patients with various illnesses and health concerns. This questionnaire comprised the same questions as the interview study and therefore the results of the two studies were analyzed together.

On top of this, more informal research methods were used, namely volunteering as a respite worker for several hours a week for 9 months throughout the study. This was primarily done to better engage and connect with the target audience and support groups.

\section{Study findings}

Interviews \& questionnaire study

The interview $(N=4)$ and questionnaire study $(N=34)$ led to 38 participants in total (age range: 17-82) from which 35 were female and 3 were male $(N=38: 14$ 
4. Caregiving book (respite care preparation) (Mean $=3.5$ / $S D=1.3$ / used by $18 \%$ ). A book in which the caregiver can record how they address the care and concern for their care recipient, to serve as a detailed guide for replacement care.

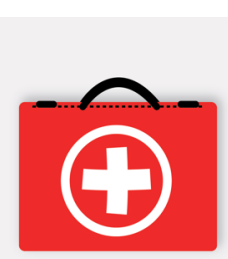

5. Goodie kit for caregivers (Mean=3.4 / SD $=1.3$ / used by $16 \%$ ). A special kit for caregivers with tools and tips about caring for someone and balancing care provision, work and life.

6. Caregiver telephone line (Mean=3.4 / SD =1.3/ used by $24 \%$ ). A telephone line that caregivers can call for information, advice or support.

7. Measuring the caregivers' health (Mean $=3.4 / \mathrm{SD}=1.4$ / used by $11 \%)$. A tool to measure and read daily stress levels to learn whether or not a caregiver is taking enough rest and time for oneself.
Dutch caregivers, 13 American, 4 Belgian, 3 Australian, 3 British and 1 Canadian). As most participants were recruited through Facebook caregiver support groups, the female participant predominance could suggest that women feel more need to use such a social forum. However, there are also typically more female than male caregivers $[12,31]$. Caregivers in the study population took care of their parent $(n=18)$ or spouse $(n=13)$ in most cases. Thirty-four caregivers were assessed with the Caregiver Strain Index, which indicated 26 of them to be overburdened. The majority provided care for over 16 hours a week $(n=21)$ and for longer than 3 years $(n=20)$, and most caregivers $(n=21)$ received (occasional) help from others.

Overall, the caregivers positively believed that a technological solution could support them $(M=3.4$, on a five-point scale, on which $1=$ poor and $5=$ excellent) however, they had more confidence in a financial solution $(M=4.4)$. Further, the interview and questionnaire study revealed that it is challenging for caregivers to prepare themselves for the future, as there are many uncertainties and the situation and needs of their care recipient can change over time. This consequently impacts the caregivers' own needs. As one participant stated: "The information I was given initially gave no indication as to what lay ahead." The majority $(n=19)$ thus learned how to help and what was needed through a process of trial and error. Almost all caregivers were worried about the future of their loved ones and whether s/he was suffering. Some caregivers stated they were especially worried when they were not with their loved one, but somewhere else. Caregivers were more focused on their care recipients and their own health was often not a priority to them: "It was easy to neglect myself and just care for dad." Some caregivers explained it was challenging to recognize when they needed to take a break. Most participants $(n=24)$ indicated that their social life had regressed since becoming a caregiver. Consistent with other studies $[7,25,26]$, caregivers also reported many positive sides of caregiving. Moments of happiness that the caregivers described were predominantly interpersonal (family) occasions, such as sharing moments of affection and surprising experiences. Also, participants pointed out that they had learned new skills through caregiving (e.g. motivating people, compassion, patience and practical skills such as cooking and personal care). Caregivers stated that they needed better information resources (e.g. on the illness of their care recipient), workshops or training about caregiving-related topics and more help from family members or other caregivers. They indicated to have trouble asking for help and were often not aware of support resources available to them. The majority of caregivers $(n=31)$ stated to frequently use online resources to look up care related information, e.g. reports and advice concerning the health issues of their care recipient. Many $(n=24)$ used online peer support groups. Some caregivers were aware of online tools such as websites for care task management, but only few $(n=6)$ pointed out to use such a service.

\section{Solution scenarios}

All proposed solution scenarios (see sidebar page 3-6) were generally reviewed as positive. There was no significant preference in choosing for patient or caregiver-centric solutions $(\mathrm{t}(16)=-1,05, p=0.31)$, nor in choosing for existing or non-existing solutions $(\mathrm{t}(15)=-0.74, p=0.47)$. There was, however, a significant positive relationship between the solutions that the participants already used and the valued mean 


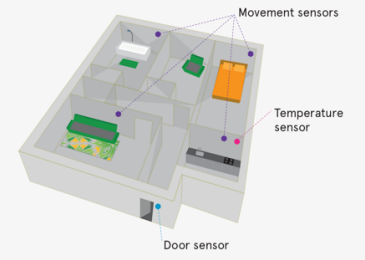

8. Sensor monitoring (Mean=3.3 / SD=1.5 / used by $16 \%)$. Monitoring the care recipient by deploying smart sensors in the home.

9. Fall alarm (Mean $=3.3$ / $\mathrm{SD}=1.5$ / used by $24 \%$ ). A necklace with a button that can trigger an alarm.

10. Outsourcing care tasks (Mean=3.2 / SD=1.6/ used by $8 \%)$. A website to help outsource small care tasks or chores to volunteers, friends and neighbors in the local community.

11. Care management (Mean=3.1 / SD=1.5 / used by $16 \%)$. An online platform to plan and manage care tasks and communicate with other caregivers.

12. House automation (Mean=3.1 / SD=1.5 / used by $11 \%$ ). Automating electronics, heating and lighting in the home of the care recipient. score $(r=0.67, p=0.003)$. For example, the most used existing solutions were network groups $(n=24)$ and online information tool $(n=23)$, while overall the most popular ranked solutions were an information tool $(M=3.9)$ followed by caregiver relaxation $(M=3.7)$ and online caregiver network groups $(M=3.6)$. These findings suggest caregivers' need for (better) information, relaxation and (social) support. The highest ranked new solutions were caregiver relaxation $(\mathrm{M}=3.7)$, measuring caregiver's health $(\mathrm{M}=3.4)$ and a goodie kit for caregivers $(M=3.4)$. The least popular scenarios were an application for communication between caregiver and patient $(\mathrm{M}=2.7)$ and care robots for companionship for care recipients $(M=2.5)$ Solutions for safety, assistance and reassurance, such as sensor monitoring, house automation and remote care ranked higher.

\section{Design opportunities}

Based on the study findings, the following design opportunities were identified:

- Increase awareness of current solutions and make them accessible, flexible and manageable: Caregivers indicated a need to be made better aware and informed on caregiving related topics and tools, as current available solutions were often unknown to them. One clear opportunity is thus to work toward solutions that make caregivers more aware of potential helpful sources when appropriate, to suit the varying and changeable care situations. Factors such as flexibility and adaptability were found important requirements for solutions in caregiving context. Furthermore, solutions should not add to the burden of the caregiver and consequently be manageable, easy to access and integral [6]. The
Goodie kit for caregivers (as described in the scenarios) could comprise a collection of tools that positively fuel and support caregivers, focusing on different aspects or phases of caring.

- Focus on caregivers' wellness: The study suggested that caregivers tend to prioritize the health of the person they care for. However, out of the new solution scenarios, the participants ranked solutions such as caregiver relaxation and measuring caregiver's health high. Solutions that thus encourage caregivers to take time for themselves, relax and make them more aware of their own health have interesting potential in helping to prevent caregiver burden.

- Positive solutions: Caregivers that shared positive stories about caregiving during the interviews stated that these experiences strongly contributed to the sense of gratification they felt. Solutions that encourage capturing, highlighting or sharing these positive moments could thus help to emphasize the positive outlook of caregivers and so relieve a felt burden.

- Strengthen social ties: As most caregivers indicated that their social life had been deteriorating, solutions that focus on meaningful and pleasant social interactions could address this issue. This finding is consistent with other studies (e.g. [27]).

- Provide reassurance: Many caregivers indicated to worry about their loved-one, especially when they were not around. Solutions that provide reassurance and confirmation that the care recipient is doing well could target this need and decrease the worry of caregivers. 


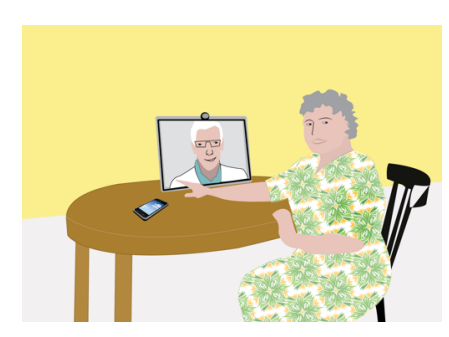

13. Remote care provision (Mean=3.1 / SD=1.4/ used by $5 \%$ ). Communicating with healthcare professionals from home through an audio/video connection.

14. Self-measurement tool for care recipient (Mean=3.1 / $\mathrm{SD}=1.4$ / used by $24 \%$ ). Using tools to measure and track the health of the care recipient at home.

15. Life book (Mean=2.8 / $\mathrm{SD}=1.6$ / used by $24 \%$ ).

Creating a book about the life of the care recipient together with family, friends and the care recipient.

16. How are you doing? (Mean=2.7 / SD=1.5 / used by $16 \%)$. An application through which the caregiver and care recipient can indicate how they are doing.

17. Care robot (Mean $=2.5$ / $\mathrm{SD}=1.4$ / used by $5 \%$ ). A robot that can keep a care recipient company, chat with them and even play games.

\section{Discussion and future work}

This paper presented a study for identifying potential technological opportunities for supporting informal caregivers. In doing this, the whole (individual, societal and changing) context of the caregiver should be considered. Although technology should not be seen as the sole solution for unburdening caregivers, the study showed that there are certainly opportunities and lessons learned in terms of designing supportive technologies for this particular target group, namely:

- Focus on the informal caregiver: Not only the care recipient is in need of supportive care and solutions; we argued that as informal caregivers have become imperative for home care provision, in the design for any technology in the context of in-home care the (emotional) needs of caregivers must be considered.

- Deploy research approaches that are integral and relieve caregivers instead of increasing the burden: For our interview study, it was challenging to recruit participants. Indeed, participating in a study typically takes time and resources. This is a particular point of concern in the case of caregivers, as this group is typically already overloaded. Consequently, not only the designed solution, but also the research method should evidently not overload the participant. Also, caregiving is a personal and delicately private affair. Caregivers in the Netherlands are usually not (formally) known or registered. Thus additionally, creative tactics - such as volunteering for informal care support groups- to better engage with in-home caregivers should be explored.

- Deploy research and design approaches that are more positive-oriented: Approaches inspired by positive psychology, e.g. the experience sampling method [11], could be promising for capturing positive moments and moving away from the negative stressors of caregiving. This novel perspective focuses on wellness (of the caregiver) instead of illness (of the care recipient).

- Be mindful of techno-solutism: The results showed that participants generally believed more in a financial solution, than a technological one. Thus, technology should not be seen as the sole solution. Still, the participants also believed that technologies can support the caregiver. The paper outlined several design opportunities to do so.

The aim of this study was to investigate whether and how technological solutions can truly contribute to unburdening informal caregivers. For this purpose, the needs of caregivers and current and potential ICT solutions were investigated to identify which aspects of these systems are desired. It is now key that such potential new solutions are studied in interactive usage and designed in close collaboration with caregivers so to best match with the surrounding context of its users. This is important because the success of work in this area depends on adequately identifying and responding to caregivers' needs, as well as understanding and incorporating appropriate strategies for technology design and development that is truly supportive.

\section{Acknowledgements}

We would like to thank all the participants in our study and the reviewers of this paper. We thank Ben Kröse, Rick Kwekkeboom, Yvette Wittenberg, Rieke Hengelaar, Julia Dunn and Kearsley Stewart for their contributions to this work. This work is part of the research projects Co-Care-IT (funded by the AUAS program Urban Vitality) and FIT (funded by TKI of the Dutch Ministry of Economic affairs) 


\section{References}

1. Ronald D. Adelman, Lyubov L. Tmanova, Diana Delgado, Sarah Dion, and Mark S. Lachs. 2014. Caregiver Burden: a Clinical Review. JAMA 311, 10: 1052.

2. Ana Correia de Barros, João Cevada, Àngels Bayés, Sheila Alcaine, and Berta Mestre. 2013. User-centred design of a mobile self-management solution for Parkinson's disease. Proceedings of the 12th International Conference on Mobile and Ubiquitous Multimedia - MUM'13, 1-10.

3. Marina Bastawrous. 2013. Caregiver burden-A critical discussion. International Journal of Nursing Studies 50, 3: 431-441.

4. Claus Bossen, Lars Rune Christensen, Erik Grönvall, and Lasse Steenbock Vestergaard. 2013. CareCoor: Augmenting the coordination of cooperative home care work. International Journal of Medical Informatics 82, 5: e189-e199.

5. Tone Bratteteig and Ina Wagner. 2013. Moving Healthcare to the Home: The Work to Make Homecare Work. In ECSCW 2013: Proceedings of the 13th European Conference on Computer Supported Cooperative Work, 21-25 September 2013, Paphos, Cyprus. Springer London, London, 143-162.

6. Yunan Chen, Victor Ngo, and Sun Young Park. 2013. Caring for Caregivers: Designing for Integrality. Proceedings of the 2013 conference on Computer supported cooperative work - CSCW '13, ACM Press, 91.

7. Carole a. Cohen, Angela Colantonio, and Lee Vernich. 2002. Positive aspects of caregiving: rounding out the caregiver experience. International Journal of Geriatric Psychiatry 17, 2: 184-188.

8. A.M. Cole and Binh Q. Tran. Home automation to promote independent living in elderly populations. Proceedings of the Second Joint 24th Annual Conference and the Annual Fall Meeting of the Biomedical Engineering Society] [Engineering in Medicine and Biology, IEEE, 2422-2423.

9. Kathryn Daniel, Carolyn L. Cason, and Sherry Ferrell.
2009. Assistive technologies for use in the home to prolong independence. Proceedings of the 2nd International Conference on PErvasive Technologies Related to Assistive Environments, 26.

10. Rie Fujisawa and Francesca Colombo. 2009. The LongTerm Care Workforce: Overview and Strategies to Adapt Supply to a Growing Demand. Paris, France.

11. Joel M. Hektner, Jennifer A. Schmidt, and Mihaly Csikszentmihalyi. 2007. Experience Sampling Method Measuring the Quality of Everyday Life. SAGE Publications, Inc, Thousand Oaks, California.

12. The National Alliance for Caregiving (NAC) and the AARP Public Policy Institute. 2015. Caregiving in the U.S. 2015.

13. Myounghoon Jeon, Nazneen Nazneen, Ozum Akanser, Abner Ayala-Acevedo, and Bruce Walker. 2012. "Listen2dRoom": Helping Blind Individuals Understand Room Layouts Abstract. Proceedings of the 2012 ACM annual conference extended abstracts on Human Factors in Computing Systems Extended Abstracts CHI EA '12, ACM Press, 1577.

14. Marije Kanis, Willem Paul Brinkman, and Mark Perry. 2009. Designing for positive disclosure: What do you like today? International Journal of Industrial Ergonomics 39, 3: 564-572.

15. Marije Kanis, Saskia Robben, Judith Hagen, Anne Bimmerman, Natasja Wagelaar, and Ben Kröse. 2013. Sensor Monitoring in the Home: Giving Voice to Elderly People. Pervasive Computing Technologies for Healthcare (PervasiveHealth), 2013 7th International Conference on, 97-100.

16. Jiro Kawakita. 1982. The Original KJ Method. Kawakita Research Institute, Tokyo.

17. Elizabeth Kaziunas, Ayse G Buyuktur, Jasmine Jones, Sung W Choi, David a Hanauer, and Mark S Ackerman. 2015. Transition and Reflection in the Use of Health Information. Proceedings of the 18th ACM Conference on Computer Supported Cooperative Work \& Social Computing - CSCW '15, ACM Press, 1763-1774.

18. Ben Kröse, Tim van Oosterhout, and Gwenn Englebienne. 2014. Video Surveillance for Behaviour 
Monitoring in Home Health Care. Proceedings of Measuring Behavior 2014.

19. Matthew L. Lee and Anind K. Dey. 2008. Using lifelogging to support recollection for people with episodic memory impairment and their caregivers. Proceedings of the 2nd International Workshop on Systems and Networking Support for Health Care and Assisted Living Environments - HealthNet '08, ACM Press, 1.

20. Janice Loh, Tomas Schietecat, Tsun Fai Kwok, Lucas Lindeboom, and Peter Joore. 2004. Technology applied to address difficulties of Alzheimer patients and their partners. Proceedings of the conference on Dutch directions in $\mathrm{HCI}$-, ACM Press, 18.

21. Maurice Mulvenna, Suzanne Martin, Stefan Sävenstedt, et al. 2010. Designing \& Evaluating a Cognitive Prosthetic for People with Mild Dementia. Proceedings of the 28th Annual European Conference on Cognitive Ergonomics - ECCE '10, ACM Press, 11.

22. Saskia Robben, Lilian Bosch, Pascal Wiggers, Jasmien Decancq, and Marije Kanis. 2015. Managing Flexible Care With a Context Aware System for Ageing-InPlace. Pervasive Health 2015, 9th International Conference on Pervasive Computing Technologies for Healthcare.

23. Betsy C. Robinson. 1983. Validation of a Caregiver Strain Index. Journal of Gerontology 38, 3: 344-348.

24. Frank Rudzicz, Rozanne Wilson, Alex Mihailidis, Elizabeth Rochon, and Carol Leonard. 2012. Communication strategies for a computerized caregiver for individuals with Alzheimer's disease. SLPAT '12 Proceedings of the Third Workshop on Speech and Language Processing for Assistive Technologies, Association for Computational Linguistics, 47-55.

25. Sally Savage and Susan Bailey. 2004. The impact of caring on caregivers' mental health: a review of the literature. Australian health review : a publication of the Australian Hospital Association 27: 111-117.

26. Hilda Tellioğlu, Myriam Lewkowicz, Aparecido Fabiano Pinatti de Carvalho, Ivan Brešković, and Marén
Schorch. 2014. Collaboration and coordination in the context of informal care. Proceedings of the companion publication of the 17th ACM conference on Computer supported cooperative work \& social computing (CSCW Companion '14)., 339-342.

27. Matthieu Tixier and Myriam Lewkowicz. 2015. Looking for Respite and Support: Technological Opportunities for Spousal Caregivers. Proceedings of the 33rd Annual ACM Conference on Human Factors in Computing Systems - CHI '15, 1155-1158.

28. Kevin C. Tseng, Chien Lung Hsu, and Yu Hao Chuang. 2013. Designing an intelligent health monitoring system and exploring user acceptance for the elderly. Journal of Medical Systems 37, 6: 9967.

29. Carers UK. 2015. Buddi. Retrieved September 26, 2015 from http://www.carersuk.org/help-andadvice/equipment-and-technology/our-products-forcarers/budd

30. Antoine A. J. van de Ven, Anne-mie A.G. Sponselee, and Ben A.M. Schouten. 2010. Robo M.D.: A Home Care Robot for Monitoring and Detection of Critical Situations. Proceedings of the 28th Annual European Conference on Cognitive Ergonomics - ECCE '10, ACM Press, 375.

31. Anders Wimo and Martin Prince. 2010. Alzheimer's Disease International - World Alzheimer Report 2010, The Global Economic Impact of Dementia. London. Retrieved from

http://www.alz.co.uk/research/files/WorldAlzheimerRe port.pdf

32. Sheng-Hsiang Yu, Li-Shan Wang, Hao-Hua Chu, et al. 2011. A mobile mediation tool for improving interaction between depressed individuals and caregivers. Personal and Ubiquitous Computing 15, 7 : 695-706. 